\title{
Angiographic Findings in Diaphragmatic Rupture
}

\author{
W. Dennis Foley, D.D.R., ${ }^{*}$ and Stewart R. Reuter, M.D. \\ From the Department of Radiology, University of Michigan, Ann Arbor, Michigan 48104
}

The incidence of traumatic rupture of the diaphragm has increased during the past decade, a reflection of the increased number of high speed automobile accidents. The left hemidiaphragm is more commonly ruptured by blunt abdominal trauma. ${ }^{1}$ Occasionally, however, a right hemidiaphragmatic rupture may allow the liver to herniate into the chest.

Rupture of the left hemidiaphragm is generally diagnosed in the emergency room from the initial clinical examination supplemented by chest and abdominal radiographs." If stomach or bowel have herniated, the radiographs usually have a characteristic appearance. However, herniation of the spleen is more difficult to establish. Accompanying splenic, hepatic, mesenteric or renal rupture is frequent, and hemothorax is not unusual. ${ }^{3}$

Diagnosis of a ruptured right hemidiaphragm may be difficult and the condition may not be suspected until the patient is recovering from other injuries. The usual roentgenographic findings are pleural effusion, basal atelectasis and an apparent elevation of the right hemidiaphragm. Since herniation of the colon and small bowel is uncommon on the right, barium studies are generally inconclusive. A pneumoperitoneum may establish the diagnosis, but occlusion of the diaphragmatic tear by the herniated structures can prevent free passage of air from the peritoneal to the pleural space....

Since the diagnosis of diaphragmatic rupture is occasionally difficult, angiography may be useful. In addition, unsuspected ruptures may be present in patients who are being evaluated for other types of visceral trauma. We are therefore presenting the angiographic abnormalities observed in four patients with diaphragmatic rupture.

\section{CASE REPORTS}

Case I. A 23 year old man was admitted to the emergency room unconscious and in shock following an automobile accident. The patient had microscopic hematuria, and abdominal lavage was positive for intraperitoneal blood.
A chest roentgenogram revealed left hemothorax and an apparent elevation of the left hemidiaphragm. The patient had several fractures, including a fractured pelvis.

Emergency thoracic and abdominal aortography were performed. The thoracic aortogram was normal; the abdominal aortogram revealed a splenic rupture. The short gastric branches of the splenic artery and the left gastric artery branches were elevated and stretched into the left hemithorax, indicating gastric herniation (Figure 1). At laparotomy, the stomach had herniated through a rupture of the left diaphragm. The ruptured spleen remained in the abdomen.

Case II. A 60 year old man was admitted to the hospital following extensive burns and a left hemothorax resulting from a fall while repairing high tension wires. A ruptured left hemidiaphragm was suspected on the initial chest roentgenograms. A thoracic aortogram revealed a normal aorta. A celiac angiogram demonstrated that the spleen was not ruptured and had not herniated through the diaphragm. However, short gastric branches of the splenic artery and left gastric artery branches were stretched into the left lower chest, indicating herniation of the stomach into the chest. These findings were confirmed at operation.

Case III. A 25 year old man was admitted to the emergency room unconscious and in shock following an automobile accident. The patient had several fractures, and peritoneal lavage revealed free intraperitoneal blood. A chest radiograph demonstrated a left hemothorax and probable rupture of the left hemidiaphragm. An emergency thoracic aortogram revealed a normal aorta; an abdominal aortogram revealed that a ruptured spleen had herniated into the lower thorax. In addition, stretching of the jejunal arteries into the left

\footnotetext{
PRESENT ADDRESS:

Department of Radiology, Sir Charles Gairdner Hospital, Perth Medical Centre, Shenton Park,
} Western Australia 6008.

Australasian Radiology, Vol. XX, No. 2, June, 1976 


\section{Angiographic Findings in Diaphragmatic Rupture}

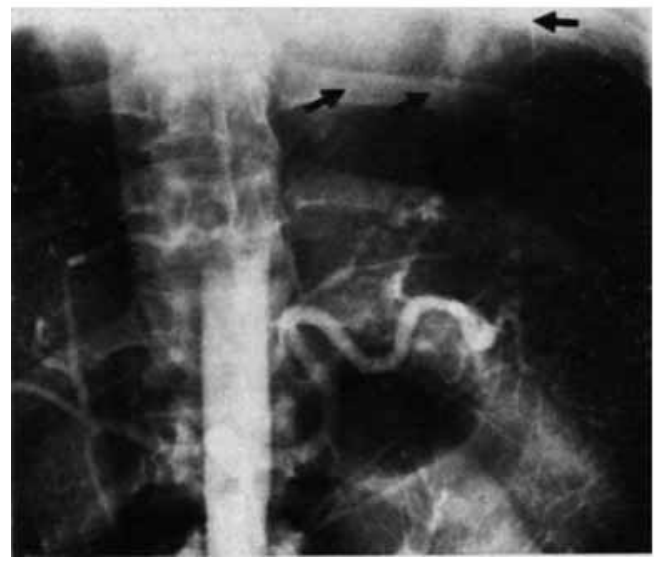

(a) Arterial phase of abdominal aortography. Branches of the left gastric and short gastric arteries extend into the chest (arrows).

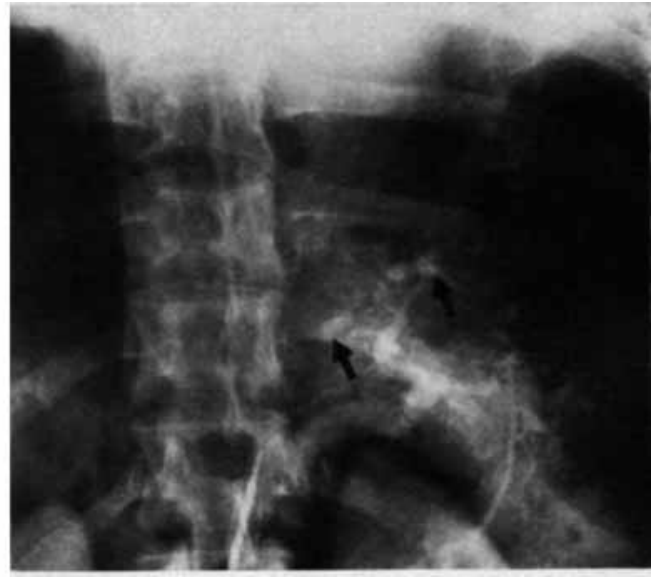

(b) Venous phase. Extravasated contrast medium in splenic lacerations indicates splenic rupture (arrows).

Figure 1-Rupture of the left hemidiaphragm in a 23 year old man.

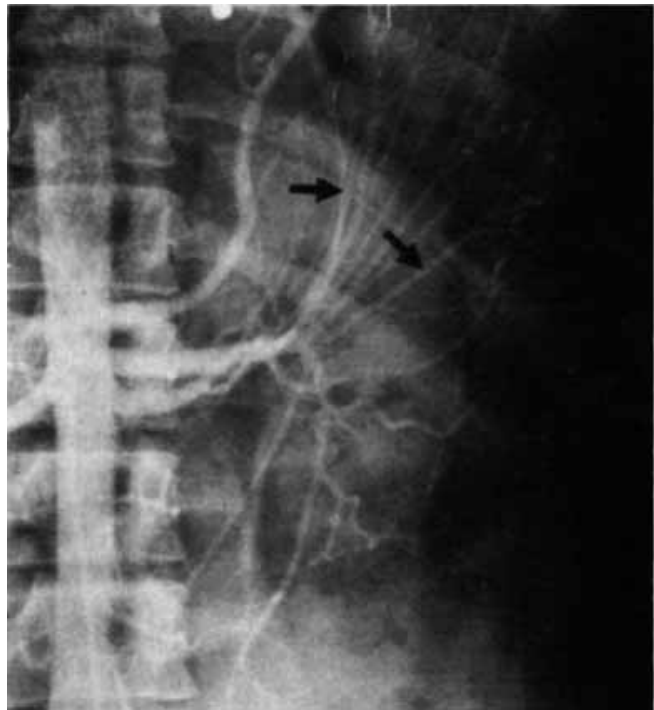

(a) Arterial phase of abdominal aortography. Jejunal branches of the superior mesenteric artery extend into the chest (arrows). The herniated splenic artery is medial to the jejunal arteries; the left gastric artery is not seen.

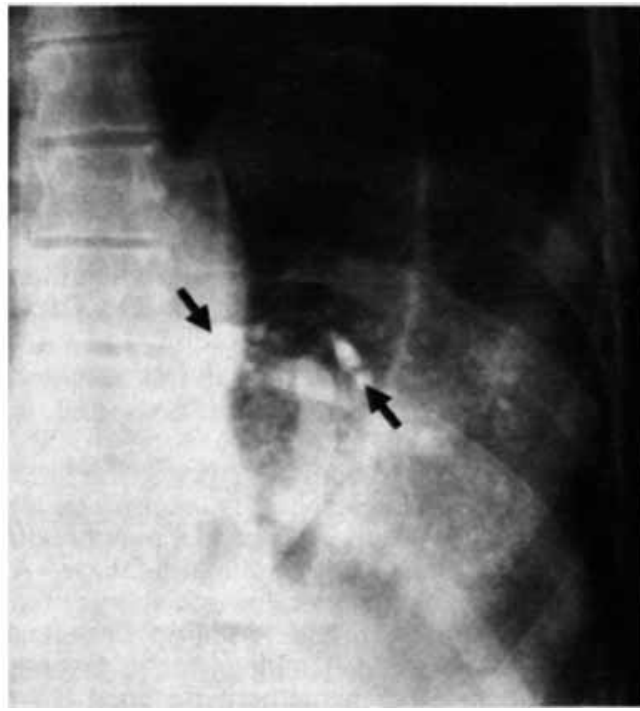

(b) Venous phase. The partially distended stomach is superimposed in the ruptured spleen. Contrast medium has extravasated into sp'enic lacerations (arrows). The splenic vein drains inferomedialiy.

FIGURE 2-Rupture of the left hemidiaphragm in a 25 year old man. 


\section{W. Dennis Foley and Stewart R. Reuter}

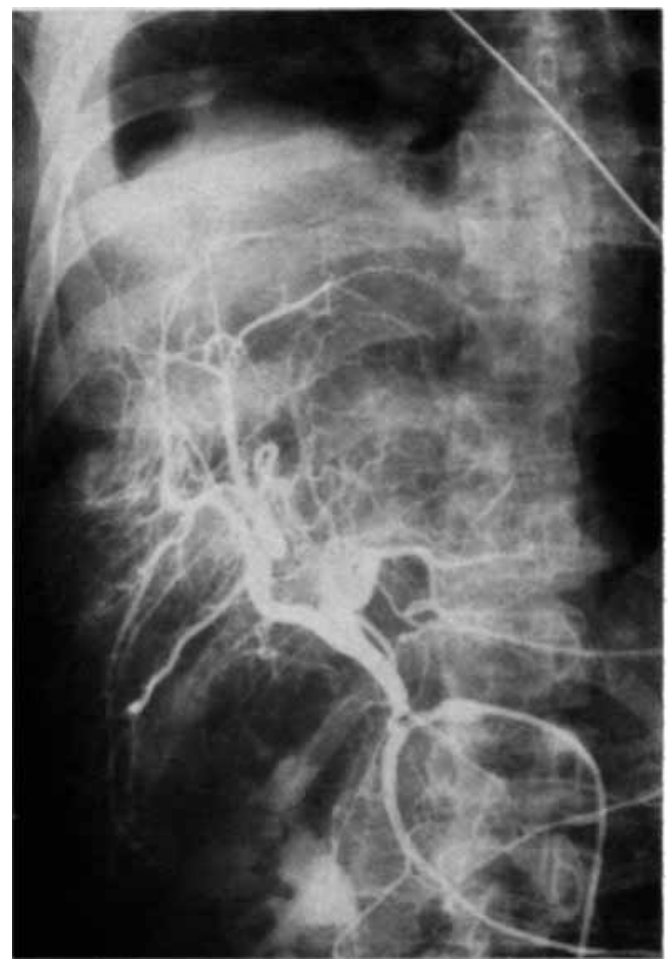

(a) Arterial phase of hepatic angiography. The hepatic artery branches are stretched and distorted, indicating contusion. No extravasation or hematoma is seen. The liver extends into the left hemithorax and a hemothorax is seen.

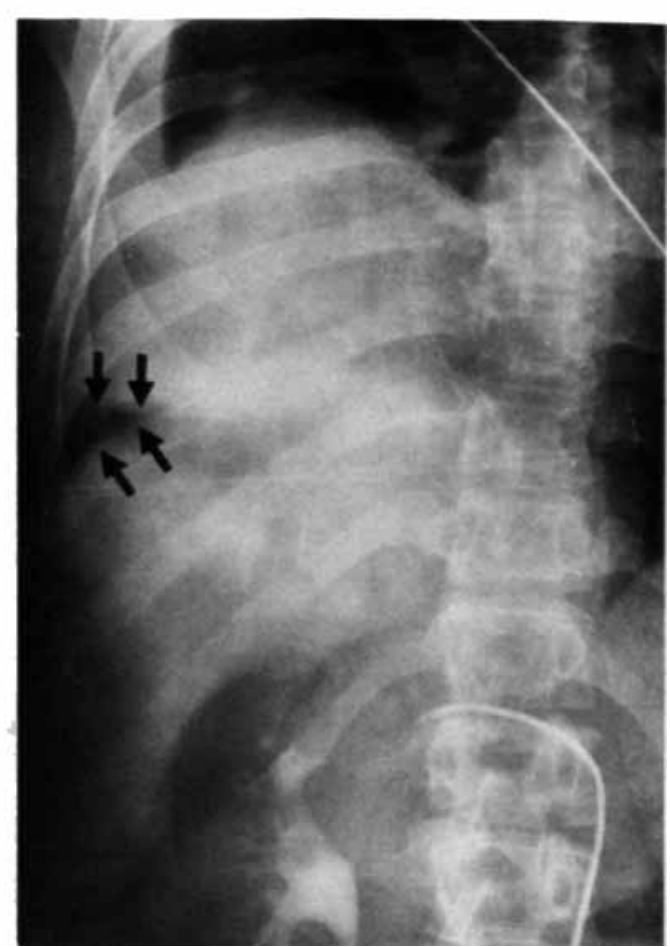

(b) Venous phase. The impression made by the edge of the diaphragm into the liver can be seen (arrows).

FiguRE 3-Rupture of the right hemidiaphragm in a 25 year old woman.

hemithorax indicated herniation of the jejunum into the left chest as well (Figure 2). These findings were confirmed at operation.

Case IV. A 25 year old woman was admitted to the emergency room unconscious and in shock following an automobile accident. She had several fractured bones, but no rib fractures were present. The respiratory excursions of the right chest were decreased and breath sounds were diminished at the right base. A chest roentgenogram revealed atelectasis and effusion at the right base with an apparent elevation of the right hemidiaphragm. Over a four day period her hematocrit dropped from 35 to $28 \%$. A hepatic angiogram revealed herniation of the liver into the right hemithorax with arterial changes of hepatic contusion (Figure 3). No bleeding site was demonstrated. The abdomen was explored, the liver reduced, and the diaphragm repaired.

\section{Discussion}

The angiographic findings in diaphragmatic rupture vary with the side of rupture and the structures that have herniated. All three of our patients with rupture of the left hemidiaphragm had gastric herniation. The short gastric and left gastric artery branches were stretched and terminal branches distributed into the lower left thorax. If the small bowel is also herniated, superior mesenteric artery branches may be similarly stretched and displaced. Splenic rupture frequently accompanies rupture of the left hemidiaphragm, but the spleen may either herniate or remain in the abdomen.

With rupture of the right hemidiaphragm, the liver is generally the only organ herniating and angiography reveals contusion and/or laceration of a liver situated partially in the chest. As in our patient, an indentation in the lateral liver margin may identify the site of the diaphragm. 
Angiography is probably more useful in evaluating patients with suspected rupture of the right hemidiaphragm, since the herniated dome of the liver may mimic an elevated diaphragm. On the left, herniation of the bowel or stomach generally makes the diagnosis apparent on conventional examinations. If a hemothorax is associated with the rupture, thoracic aortography may be required to exclude aortic rupture. Abdominal aortography or selective visceral angiography performed at the same time as the thoracic aortogram may confirm the presence of the diaphragmatic rupture, reveal the organs which have herniated, and rule out other visceral injuries.

\section{SUMMARY}

The angiographic findings are presented in four patients with ruptured diaphragms. With rupture of the left hemidiaphragm the findings vary with the structures herniating. Gastric, mesenteric or splenic artery branches extending into the lower chest indicate herniation of the stomach, bowel or spleen. A herniating spleen is frequently ruptured. On the right, the liver is generally the only organ to herniate. An indentation in the lateral liver margin in the venous phase, if present, indicates the level of the diaphragm.

\section{REFERENCES}

${ }^{1}$ Bekassy, S. M., Dave, K. S., Wooler, G. H., and Ionescu, M. I. (1973): "Spontaneous' and Traumatic Rupture of the Diaphragm: Long-term Results." Ann. Surg., 177, 320.

${ }^{2}$ Lavender, J. P., and Potts, D. G. (1959): "Differential Diagnosis of Elevated Right Diaphragmatic Dome." Brit.J. Radiol. 32, 56.

${ }^{3}$ Strug, B., Noon, G. P., and Beall, A. C., Jr. (1974): "Traumatic Diaphragmatic Hernia." Ann. Thor. Surg., 17, 444.

\section{Midwinter Radiological Conference of the Los Angeles Radiological Society}

The Los Angeles Radiological Society will hold its 29th Annual Midwinter Radiological Conference on January 28th, 29th and 30th, 1977, at the Century Plaza Hotel in Los Angeles. The first day of the meeting is devoted to small size workshops. Separate didactic sessions in diagnosis and radiotherapy by an outstanding faculty will be held the last two days. The country's second largest technical exhibit will be open for viewing all three days.
An optional post-Conference Hawaiian Seminar combining teaching and relaxation will again be offered.

For further information, please contact:
Dr. Larry Kussin,
Secretary-Treasurer, 15107 Vanowen Street, Van Nuys, California 91405 , U.S.A. 